\title{
An HDG formulation for incompressible and immiscible two-phase porous media flow problems
}

\author{
A. Costa-Soléa, E. Ruiz-Gironés ${ }^{\mathrm{b}}$, J. Sarrate $^{\mathrm{a}}$ \\ ${ }^{a}$ Laboratori de Càlcul Numric (LaCàN), Universitat Politècnica de Catalunya, Building C2, \\ Campus Nord UPC, Jordi Girona 1, 08034-Barcelona (SPAIN); \\ b Barcelona Supercomputing Center - BSC, Building Nexus II, Jordi Girona, 29-31, 08034 \\ Barcelona (SPAIN)
}

\section{ARTICLE HISTORY}

Compiled May 16, 2019

\begin{abstract}
We develop a high-order hybridizable discontinuous Galerkin (HDG) formulation to solve the immiscible and incompressible two-phase flow problem in a heterogeneous porous media. The HDG method is locally conservative, has fewer degrees of freedom than other discontinuous Galerkin methods due to the hybridization procedure, provides built-in stabilization for arbitrary polynomial degrees and, if the error of the temporal discretization is low enough, the pressure, the saturation and their fluxes converge with order $P+1$ in $L^{2}$-norm, being $P$ the polynomial degree. In addition, an element-wise post-process can be applied to obtain a convergence rate of $P+2$ in $L^{2}$-norm for the scalar variables. All of these advantages make the HDG method suitable for solving multiphase flow trough porous media. We show numerical evidence of the convergences rates. Finally, to assess the capabilities of the proposed formulation, we apply it to several cases of water-flooding technique for oil recovery.
\end{abstract}

\section{KEYWORDS}

Two-phase flow; immiscible; incompressible; hybridizable discontinuous Galerkin method; diagonally implicit Runge-Kutta method; differential algebraic equations.

\section{Introduction}

Nowadays, numerical simulation of oil reservoirs plays a major role in petroleum engineering. During the initial stages of hydrocarbon production (primary recovery), the pressure difference between the reservoir and the separator at the surface is high enough to move the hydrocarbons, see Donaldson, Chilingarian, and Yen (1985). Afterwards, as the pressure of the reservoir decreases, a fluid (usually water) is injected to maintain the flow rate. This is known as secondary recovery, see Donaldson, Chilingarian, and Yen (1985). If a single hydrocarbon is considered and the pressure is above the bubble point, the two-phase immiscible flow model is widely used in industry to simulate this process, see Chen, Huan, and Ma (2006).

To obtain accurate approximations of the pressure, the saturation and the Darcy velocity of each phase, several requirements have to be fulfilled. The formulation has to 
deal with unstructured meshes to capture heterogeneous and complex subsurface configurations. Moreover, it has to provide high-accurate solutions without hampering the computational cost. Therefore, unstructured high-order formulations are well-suited for these applications. In addition, the formulation has to be stable and the mass should be conserved, at least, at the element level. Nowadays, several methods such as the finite differences (FD), finite volumes (FV), continuous finite elements (CG), mixed finite elements (Mixed CG), have been applied. While all of these methods have their own advantages, none of them totally fulfill previous requirements. Recently, discontinuous Galerkin methods (DG) have been shown as competitive discretization methods in these type of problems, see Klieber and Rivière (2006); Epshteyn and Rivière (2007). DG methods approximate the scalar variable $u$ as well as the flux variable $\mathbf{q}$, related to $\boldsymbol{\nabla} u$, using discontinuous element-wise polynomials of arbitrary degree. Each DG formulation imposes the weak continuity of the solution in a different manner. Several advantages arise from using DG methods. For instance, mass conservation is verified at the element level, and they allow using any polynomial degree. DG methods can improve the accuracy of the computed approximations because they can use polynomials of arbitrary degree, see Kirby, Sherwin, and Cockburn (2012). In order to increase the computational efficiency several DG methods can be restated in the so called primal formulation where the vector flux variable is replaced by its expression in terms of the main unknown $u$, thereby significantly reducing the number of unknowns. This formulation is used in Klieber and Rivière (2006) to solve the two-phase flow problem.

In this paper, we apply a high-order hybridizable discontinuous Galerkin (HDG) formulation for solving two-phase flow through porous media. HDG uses the scalar unknown, $u$, and the flux, q, as DG method does, see Kirby, Sherwin, and Cockburn (2012); Nguyen, Peraire, and Cockburn (2009a,b, 2011). The HDG formulation imposes the continuity of the normal component of a numerical flux between elements.

The boundary value problem is stated element-by-element and continuity of the solution $\mathrm{u}$ is imposed in weak form, by introducing a new variable on the mesh skeleton, $\hat{u}$, which is the trace of $u$. The HDG method provides built-in stabilization for any polynomial degree and ensures element-wise mass balance. Moreover, HDG uses polynomials of arbitrary degree, and it obtains a convergence rate of $P+1$ in the $L^{2}$ norm for $u$ and $\mathbf{q}$, when element-wise polynomials of degree $P \geq 0$ are considered, and the time discretization error is low enough, see Kirby, Sherwin, and Cockburn (2012); Nguyen, Peraire, and Cockburn (2009a,b); Sevilla and Huerta (2016). Furthermore, doing an element-wise post-processing the scalar unknown, $u$, converges with a rate of $P+2$ in the $L^{2}$-norm. Finally, the method can be hybridized in terms of $\hat{u}$ to reduce the computational cost.

We use an implicit and high-order temporal integration scheme that allows using large time steps without hampering the accuracy. This leads to an algebraic non-linear problem, which we solve using a fix-point iterative method that alternatively solves for the pressure and saturation until convergence. The proposed method differs from the classical IMPES method, since it solves explicitly the saturation and implicitly the pressure.

The outline of this paper is as follows. In Section 2, we introduce the governing equations of the two-phase flow problem. Then, in Section 3, we deduce the weak form associated with the HDG formulation. In Section 4, we describe the time discretization scheme used for the proposed formulation. In Section 5, we detail the proposed non-linear solver. In Section 6, we introduce the element-wise post-processing for the pressure and the saturation. In Section 7, we validate our formulation with a numerical evidence and we apply our formulation to simulate two cases of water-flooding 
technique for oil recovery in two 2D problems. Finally, in Section 8, we summarize the main contributions of this paper and we describe the issues that will be addressed in the near future.

\section{Governing equations}

The two-phase flow through porous media is characterised by a wetting phase, $w$, and the non-wetting phase, $o$. In this work, we consider water for the wetting phase and oil for the non-wetting phase. Both phases completely fill the voids of the soil, see Chen, Huan, and Ma (2006); Bear and Verruijt (2012), and verify:

$$
S_{w}+S_{o}=1,
$$

where $S_{w}$ and $S_{o}$ are the saturation for the water and oil, respectively. Due to the interface tension between phases, there is a discontinuity in the pressure field, which is the capillary pressure, $p_{c}$ :

$$
p_{c}=p_{o}-p_{w},
$$

where $p_{o}$ is the oil pressure and $p_{w}$ is the water pressure.

The governing equations for two-phase flow through porous media are provided by the mass conservation for each phase:

$$
\frac{\partial\left(\phi \rho_{\alpha} S_{\alpha}\right)}{\partial t}+\nabla \cdot\left(\rho_{\alpha} \mathbf{u}_{\alpha}\right)=\rho_{\alpha} f_{\alpha} \quad \alpha=w, o
$$

where $\phi$ is the porosity of the porous media, and $\rho_{\alpha}, \mathbf{u}_{\alpha}$ and $f_{\alpha}$ are the density the velocity and the source term of phase $\alpha$, respectively. The velocity of each phase, $\mathbf{u}_{\alpha}$, is given by the Darcy's law as:

$$
\mathbf{u}_{\alpha}=-\lambda_{\alpha} \mathbf{K} \nabla p_{\alpha} \quad \alpha=w, o,
$$

where $\mathbf{K}=\operatorname{diag}\left(k_{11}, k_{22}, k_{33}\right)$ is the absolute permeability and $\lambda_{\alpha}=k_{r \alpha} / \mu_{\alpha}$ is the phase mobility, being $k_{r \alpha}$ and $\mu_{\alpha}$ the relative permeability and the viscosity of phase $\alpha$, respectively.

There are several formulations to solve the two-phase flow problem. In this work, we use the water pressure, $p_{w}$, and the oil saturation, $S_{o}$, as main unknowns. According to Chen, Huan, and Ma (2006); Bear and Verruijt (2012), the total phase mobility and the total velocity are defined as:

$$
\begin{aligned}
\lambda_{t} & =\lambda_{o}+\lambda_{w} \\
\mathbf{u}_{t} & =\mathbf{u}_{o}+\mathbf{u}_{w} .
\end{aligned}
$$

Assuming immiscible and incompressible fluids and incompressible rock, we obtain a system of governing equations composed of the water pressure equation:

$$
-\boldsymbol{\nabla} \cdot\left(\mathbf{K}\left(\lambda_{o} \boldsymbol{\nabla} p_{c}+\lambda_{t} \boldsymbol{\nabla} p_{w}\right)\right)=f_{o}+f_{w},
$$


and the oil saturation equation:

$$
\phi \frac{\partial S_{o}}{\partial t}-\nabla \cdot\left(\lambda_{o} \mathbf{K}\left(\nabla p_{c}+\nabla p_{w}\right)\right)=f_{o}
$$

see Klieber and Rivière (2006) for additional details. In this work, the capillary pressure, $p_{c}$, and the relative permeabilities of each phase, $k_{r \alpha}$, are computed from the Brooks-Corey model, see Corey (1964):

$$
\left\{\begin{aligned}
p_{c} & =p_{e}\left(1-S_{e o}\right)^{-1 / \theta} \\
k_{r w} & =\left(1-S_{e o}\right)^{(2+3 \theta) / \theta} \\
k_{r o} & =S_{e o}^{2}\left(1-\left(1-S_{e o}\right)^{(2+\theta) / \theta}\right)
\end{aligned}\right.
$$

where $p_{e}$ is the entry pressure, $\theta$ is the pore size distribution, and

$$
S_{e o}=\frac{S_{o}-S_{r o}}{1-S_{r w}-S_{r o}}
$$

is the effective oil saturation, being $S_{r o}$ and $S_{r w}$ the residual oil and water saturations, respectively.

We consider a domain $\Omega$ and time interval $T=\left(0, t_{\text {end }}\right)$. The boundary of $\Omega$ is divided in three disjointed parts such that $\partial \Omega=\Gamma_{\text {in }} \cup \Gamma_{\text {out }} \cup \Gamma_{n f}$, where $\Gamma_{\text {in }}$ is the inflow boundary (water is injected), $\Gamma_{\text {out }}$ is the outflow boundary (water and oil are extracted) and $\Gamma_{n f}$ is the no flow boundary. Completing Equations (2) and (3) with appropriate boundary conditions, we end up with a system of two coupled non-linear partial differential equations (PDEs). On the one hand, the water pressure equation is:

$$
\left\{\begin{aligned}
-\boldsymbol{\nabla} \cdot\left(\lambda_{t} \mathbf{K} \nabla p_{w}+\lambda_{o} \mathbf{K} \nabla p_{c}\right) & =f_{o}+f_{w} & & \forall(\mathbf{x}, t) \in(\Omega, T), \\
p_{w} \Gamma_{\text {in }} & =g_{D p}^{i n} & & \forall(\mathbf{x}, t) \in\left(\Gamma_{\text {in }}, T\right), \\
p_{w}{ }^{\Gamma_{\text {out }}} & =g_{D p}^{\text {out }} & & \forall(\mathbf{x}, t) \in\left(\Gamma_{\text {out }}, T\right), \\
\mathbf{u}_{t} \cdot \mathbf{n} & =0 & & \forall(\mathbf{x}, t) \in\left(\Gamma_{n f}, T\right),
\end{aligned}\right.
$$

where $g_{D p}^{i n}, g_{D p}^{\text {out }}$ are the values of the Dirichlet boundaries condition for the pressure on the inflow and outflow boundaries, respectively. On the other hand, the oil saturation equation is:

$$
\left\{\begin{aligned}
\phi \frac{\partial S_{o}}{\partial t}-\boldsymbol{\nabla} \cdot\left(\lambda_{o} \mathbf{K}\left(\boldsymbol{\nabla} p_{c}+\boldsymbol{\nabla} p_{w}\right)\right) & =f_{o} & & \forall(\mathbf{x}, t) \in(\Omega, T), \\
S_{o}^{\Gamma_{i n}} & =g_{D s}^{\text {in }} & & \forall(\mathbf{x}, t) \in\left(\Gamma_{\text {in }}, T\right), \\
\left(\frac{\lambda_{o} \lambda_{w}}{\lambda_{t}} \mathbf{K} \nabla p_{c}\right) \cdot \mathbf{n} & =g_{N s}^{\text {out }} & & \forall(\mathbf{x}, t) \in\left(\Gamma_{\text {out }}, T\right), \\
\mathbf{u}_{o} \cdot \mathbf{n} & =0 & & \forall(\mathbf{x}, t) \in\left(\Gamma_{n f}, T\right), \\
S_{o}(\cdot, 0) & =S_{i} & & \forall \mathbf{x} \in \Omega,
\end{aligned}\right.
$$

where $g_{D s}^{\text {in }}, g_{D s}^{\text {out }}$ are the Dirichlet boundary condition values of the saturation for the inflow and outflow boundary respectively, and $g_{N s}^{\text {out }}$ is the value of the Neumann boundary condition at the output boundaries. We rewrite equations (5) and (6) as a system 
of first order PDEs by using the diffusive fluxes:

$$
\begin{aligned}
& \mathbf{q}_{p}=-\lambda_{t} \mathbf{K} \nabla p_{w} \\
& \mathbf{q}_{s}=-\lambda_{o} \mathbf{K} \nabla p_{c}
\end{aligned}
$$

see Kirby, Sherwin, and Cockburn (2012); Nguyen, Peraire, and Cockburn (2009a,b):

$$
\left\{\begin{aligned}
\boldsymbol{\nabla} \cdot\left(\mathbf{q}_{p}+\mathbf{q}_{s}\right) & =f_{o}+f_{w} & & \forall(\mathbf{x}, t) \in(\Omega, T), \\
\mathbf{q}_{p}+\lambda_{t} \mathbf{K} \nabla p_{w} & =\mathbf{0} & & \forall(\mathbf{x}, t) \in(\Omega, T), \\
p_{w} \Gamma_{\text {in }} & =g_{D p}^{\text {in }} & & \forall(\mathbf{x}, t) \in\left(\Gamma_{\text {in }}, T\right), \\
p_{w} \Gamma_{\text {out }} & =g_{D p}^{\text {out }} & & \forall(\mathbf{x}, t) \in\left(\Gamma_{\text {out }}, T\right), \\
\mathbf{u}_{t} \cdot \mathbf{n} & =0 & & \forall(\mathbf{x}, t) \in\left(\Gamma_{n f}, T\right), \\
\phi \frac{\partial S_{o}}{\partial t}+\nabla \cdot\left(\mathbf{q}_{s}+\frac{\lambda_{o}}{\lambda_{t}} \mathbf{q}_{p}\right) & =f_{o} & & \forall(\mathbf{x}, t) \in(\Omega, T), \\
\mathbf{q}_{s}+\lambda_{o} \mathbf{K} \nabla p_{c} & =\mathbf{0} & & \forall(\mathbf{x}, t) \in(\Omega, T), \\
S_{o} \Gamma_{\text {in }} & =g_{D s}^{\text {in }} & & \forall(\mathbf{x}, t) \in\left(\Gamma_{\text {in }}, T\right), \\
\left(\frac{\lambda_{o} \lambda_{w}}{\lambda_{t}} \mathbf{K} \nabla p_{c}\right) \cdot \mathbf{n} & =g_{N s}^{\text {out }} & & \forall(\mathbf{x}, t) \in\left(\Gamma_{\text {out }}, T\right), \\
\mathbf{u}_{o} \cdot \mathbf{n} & =0 & & \forall(\mathbf{x}, t) \in\left(\Gamma_{n f}, T\right), \\
S_{o}(\cdot, 0) & =S_{i} & & \forall \mathbf{x} \in \Omega,
\end{aligned}\right.
$$

\section{Weak form}

We discretize the domain, $\Omega$, with a tessellation, $\mathrm{T}_{h}$, composed of a set of elements, $e$, of polynomial degree $P$. Afterwards, we introduce the discontinuous finite element spaces associated with the tessellation, $\mathrm{T}_{h}$ :

$$
\begin{aligned}
\mathbb{V}_{h}^{P} & =\left\{v \in L^{2}\left(\Omega^{d}\right) \mid v_{\mid e} \in\left(\mathbb{S}^{P}(e)\right) \forall e \in \mathrm{T}_{h}\right\}, \\
\mathbf{W}_{h}^{P} & =\left\{\mathbf{w} \in\left(L^{2}\left(\Omega^{d}\right)\right)^{d} \mid \mathbf{w}_{\mid e} \in\left(\mathbb{S}^{P}(e)\right)^{d} \forall e \in \mathrm{T}_{h}\right\}, \\
\mathbb{M}_{h}^{P} & =\left\{\psi \in L^{2}\left(\Sigma_{h}\right) \mid \psi_{\mid f} \in\left(\mathbb{S}^{P}(f)\right) \forall f \in \Sigma_{h}\right\} .
\end{aligned}
$$

where $\mathbb{S}^{P}$ is the space of the polynomials of degree at most $P$ for triangles and tetrahedra (usually denoted by $\mathbb{P}^{P}$ ), or the tensor products of polynomials of degree at most $P$ in each coordinate direction for tensor product elements (usually denoted by $\mathbb{Q}^{P}$ ), $d$ is the space dimension and $\Sigma_{h}$ is the skeleton of the mesh, that is the set of all the element faces, $f$. We define $\mathbb{M}_{h}^{P}\left(g_{D}\right)=\left\{\psi \in \mathbb{M}_{h}^{P} \mid \psi=\Pi\left(g_{D}\right)\right.$ on $\left.\Gamma_{D}\right\}$, where $\Pi(\cdot)$ is a projection operator to the space $\left\{\left.\psi\right|_{\Gamma_{D}} \forall \psi \in \mathbb{M}_{h}^{P}\right\}$. In this work, we use a fixed 
polynomial degree for all the elements. We also define the scalar products:

$$
\begin{aligned}
(u, v)_{e} & =\int_{e} u v \mathrm{~d} \Omega \quad \forall u, v \in \mathbb{V}_{h}^{P}, \\
(\mathbf{q}, \mathbf{w})_{e} & =\int_{e} \mathbf{q} \cdot \mathbf{w} \mathrm{d} \Omega \quad \forall \mathbf{q}, \mathbf{w} \in \mathbf{W}_{h}^{P}, \\
\langle\hat{u}, \psi\rangle_{\partial e} & =\int_{\partial e} \hat{u} \psi \mathrm{d} \Gamma \quad \forall \hat{u}, \lambda \in \mathbb{M}_{h}^{P} .
\end{aligned}
$$

The HDG formulation for the water pressure corresponding to Equation (7) seeks an approximation $\left(p_{w_{h}}, \mathbf{q}_{p_{h}}, \hat{p}_{w_{h}}\right) \in \mathbb{V}_{h}^{P} \times \mathbf{W}_{h}^{P} \times \mathbb{M}_{h}^{P}\left(g_{D}\right)$ such that:

$$
\begin{aligned}
\sum_{e}\left(-\left(\boldsymbol{\nabla} v, \mathbf{q}_{p_{h}}+\mathbf{q}_{s_{h}}\right)_{e}+\left\langle v,\left(\hat{\mathbf{q}}_{p_{h}}+\hat{\mathbf{q}}_{s_{h}}\right) \cdot \mathbf{n}\right\rangle_{\partial e}\right) & =\sum_{e}\left(v,\left(f_{o}+f_{w}\right)\right)_{e} \\
\sum_{e}\left(\left(\mathbf{w}, \mathbf{A}_{p_{h}}^{-1} \mathbf{q}_{p_{h}}\right)_{e}-\left(\boldsymbol{\nabla} \cdot \mathbf{w}, p_{w_{h}}\right)_{e}+\left\langle\mathbf{w} \cdot \mathbf{n}, \hat{p}_{w_{h}}\right\rangle_{\partial e}\right) & =0 \\
\sum_{e}\left\langle\psi,\left(\hat{\mathbf{q}}_{p_{h}}+\hat{\mathbf{q}}_{s_{h}}\right) \cdot \mathbf{n}\right\rangle_{\partial e} & =0
\end{aligned}
$$

for all $(v, \mathbf{w}, \psi) \in \mathbf{W}_{h}^{P} \times \mathbb{V}_{h}^{P} \times \mathbb{M}_{h}^{P}(0)$, where $\mathbf{A}_{p}^{-1}=\mathbf{K}^{-1} / \lambda_{t}$, and

$$
\hat{\mathbf{q}}_{p_{h}}=\mathbf{q}_{p_{h}}+\tau_{p}\left(p_{w_{h}}-\hat{p}_{w_{h}}\right) \cdot \mathbf{n}
$$

is the numerical flux for the water pressure, being $\tau_{p}$ a stabilization function for the water pressure.

The HDG formulation for the oil saturation corresponding to Equation (7) seeks an approximation $\left(S_{o_{h}}, \mathbf{q}_{s_{h}}, \hat{S}_{o h}\right) \in \mathbb{V}_{h}^{P} \times \mathbf{W}_{h}^{P} \times \mathbb{M}_{h}^{P}\left(g_{D}\right)$ such that:

$$
\begin{aligned}
\sum_{e}\left(\left(v, \phi \frac{\partial S_{o_{h}}}{\partial t}\right)_{e}-\right. & \left.\left(\boldsymbol{\nabla} v, \mathbf{q}_{s_{h}}+\frac{\lambda_{o}}{\lambda_{t}} \mathbf{q}_{p_{h}}\right)_{e}+\left\langle v,\left(\hat{\mathbf{q}}_{s_{h}}+\frac{\hat{\lambda}_{o}}{\hat{\lambda}_{t}} \hat{\mathbf{q}}_{p_{h}}\right) \cdot \mathbf{n}\right\rangle_{\partial e}\right)=\sum_{e}\left(v, f_{o}\right)_{e} \\
\sum_{e}\left(\left(\mathbf{w}, \mathbf{A}_{s_{h}}^{-1} \mathbf{q}_{s_{h}}\right)_{e}-\left(\boldsymbol{\nabla} \cdot \mathbf{w}, S_{o_{h}}\right)_{e}+\left\langle\mathbf{w} \cdot \mathbf{n}, \hat{S}_{o_{h}}\right\rangle_{\partial e}\right) & =0 \\
\sum_{e}\left(\left\langle\psi,\left(\hat{\mathbf{q}}_{s_{h}}+\frac{\hat{\lambda}_{o}}{\hat{\lambda}_{t}} \hat{\mathbf{q}}_{p_{h}}\right) \cdot \mathbf{n}\right\rangle_{\partial e}\right)-\left\langle\psi, g_{N s}^{\text {out }}\right\rangle_{\Gamma_{N}^{s}} & =0
\end{aligned}
$$

for all $(v, \mathbf{w}, \psi) \in \mathbb{V}_{h}^{P} \times \mathbf{W}_{h}^{P} \times \mathbb{M}_{h}^{P}(0)$, where $\mathbf{A}_{s}^{-1}=\mathbf{K}^{-1} /\left(\lambda_{o} p_{c}{ }^{\prime}\right)$, being $p_{c}{ }^{\prime}$ the derivative of the capillary pressure respect to the oil saturation, $\hat{\lambda}_{o}$ and $\hat{\lambda}_{t}$ are the oil phase mobility and the total phase mobility evaluated using the traces, respectively, and

$$
\hat{\mathbf{q}}_{s_{h}}=\mathbf{q}_{s_{h}}+\tau_{s}\left(S_{o_{h}}-\hat{S}_{o_{h}}\right) \cdot \mathbf{n}
$$

is the numerical flux for the oil saturation, being $\tau_{s}$ a stabilization function for the oil saturation.

According to Nguyen, Peraire, and Cockburn (2009a,b), we set the diffusive stabi- 
lization parameter of Equations(9) and (11) as:

$$
\begin{gathered}
\tau_{p}=\frac{\hat{\lambda}_{t} \gamma_{\mathbf{K}}}{l_{p}}, \\
\tau_{s}=\frac{\hat{\lambda}_{o} \gamma_{\mathbf{K}}}{l_{s}},
\end{gathered}
$$

respectively, where $\gamma_{\mathbf{K}}$ is the maximum eigenvalue of the permeability matrix, $\mathbf{K}, l_{p}$ is the characteristic length for the pressure and $l_{s}$ is the characteristic length for the saturation.

We highlight that the Dirichlet boundary conditions are applied as follows:

$$
\hat{p}_{w_{h}}=\Pi\left(g_{D p}\right) \quad \forall \mathbf{x} \in \partial \mathrm{T}_{h \Gamma_{D}}, \quad \hat{S}_{o_{h}}=\Pi\left(g_{D s}\right) \quad \forall \mathbf{x} \in \partial \mathrm{T}_{h \Gamma_{D}},
$$

where $\partial \mathrm{T}_{h \Gamma_{D}}$ is the set of mesh faces on the Dirichlet boundary.

Inserting Equation (9) into Equations (8), the HDG formulation for the water pressure consists on seeking an approximation $\left(p_{w h}, \mathbf{q}_{p_{h}}, \hat{p}_{w_{h}}\right) \in \mathbb{V}_{h}^{P} \times \mathbf{W}_{h}^{P} \times \mathbb{M}_{h}^{P}\left(g_{D}\right)$ such that:

$$
\begin{aligned}
\sum_{e}\left(-\left(\boldsymbol{\nabla} v, \mathbf{q}_{p_{h}}+\mathbf{q}_{s_{h}}\right)_{e}+\left\langle v, \mathbf{q}_{p_{h}} \cdot \mathbf{n}+\tau_{p}\left(p_{w h}-\hat{p}_{w_{h}}\right)\right\rangle_{\partial e}\right)+ & \\
\sum_{e}\left(\left\langle v, \mathbf{q}_{s_{h}} \cdot \mathbf{n}+\tau_{s}\left(S_{o_{h}}-\hat{S}_{O_{h}}\right)\right\rangle_{\partial e}\right) & =\sum_{e}\left(v,\left(f_{o}+f_{w}\right)\right)_{e} \\
\sum_{e}\left(\left(\mathbf{w}, \mathbf{A}_{p_{h}}^{-1} \mathbf{q}_{p_{h}}\right)_{e}-\left(\boldsymbol{\nabla} \cdot \mathbf{w}, p_{w_{h}}\right)_{e}+\left\langle\mathbf{w} \cdot \mathbf{n}, \hat{p}_{w_{h}}\right\rangle_{\partial e}\right) & =0 \\
\sum_{e}\left\langle\psi, \mathbf{q}_{p_{h}} \cdot \mathbf{n}+\tau_{p}\left(p_{w h}-\hat{p}_{w_{h}}\right)+\mathbf{q}_{s_{h}} \cdot \mathbf{n}+\tau_{s}\left(S_{o_{h}}-\hat{S}_{o_{h}}\right)\right\rangle_{\partial e} & =0
\end{aligned}
$$

for all $(v, \mathbf{w}, \psi) \in \mathbb{V}_{h}^{P} \times \mathbf{W}_{h}^{P} \times \mathbb{M}_{h}^{P}(0)$.

Inserting Equation (11) into Equations (10), the HDG formulation for the oil saturation consists on seeking an approximation $\left(S_{o_{h}}, \mathbf{q}_{s_{h}}, \hat{S}_{o h}\right) \in \mathbb{V}_{h}^{P} \times \mathbf{W}_{h}^{P} \times \mathbb{M}_{h}^{P}\left(g_{D}\right)$ such that:

$$
\begin{gathered}
\sum_{e}\left(\left(v, \phi \frac{\partial S_{o_{h}}}{\partial t}\right)_{e}-\left(\boldsymbol{\nabla} v, \mathbf{q}_{s_{h}}+\frac{\lambda_{o}}{\lambda_{t}} \mathbf{q}_{p_{h}}\right)_{e}\right)+ \\
\sum_{e}\left(\left\langle v, \mathbf{q}_{s_{h}} \cdot \mathbf{n}+\tau_{s}\left(S_{o_{h}}-\hat{S}_{o_{h}}\right)+\frac{\hat{\lambda}_{o}}{\hat{\lambda}_{t}}\left(\mathbf{q}_{p_{h}} \cdot \mathbf{n}+\tau_{p}\left(p_{w h}-\hat{p}_{w_{h}}\right)\right)\right\rangle_{\partial e}\right)=\sum_{e}\left(v, f_{o}\right)_{e}, \\
\sum_{e}\left(\left(\mathbf{w}, \mathbf{A}_{s_{h}}^{-1} \mathbf{q}_{s_{h}}\right)_{e}-\left(\boldsymbol{\nabla} \cdot \mathbf{w}, S_{o_{h}}\right)_{e}+\left\langle\mathbf{w} \cdot \mathbf{n}, \hat{S}_{o_{h}}\right\rangle_{\partial e}\right)=0 \\
\sum_{e}\left(\left\langle\psi, \mathbf{q}_{s_{h}} \cdot \mathbf{n}+\tau_{s}\left(S_{o_{h}}-\hat{S}_{o_{h}}\right)+\frac{\hat{\lambda}_{o}}{\hat{\lambda}_{t}}\left(\mathbf{q}_{p_{h}} \cdot \mathbf{n}+\tau_{p}\left(p_{w h}-\hat{p}_{w_{h}}\right)\right)\right\rangle_{\partial e}\right)
\end{gathered}
$$

for all $(v, \mathbf{w}, \psi) \in \mathbb{V}_{h}^{P} \times \mathbf{W}_{h}^{P} \times \mathbb{M}_{h}^{P}(0)$.

Let $\left\{N_{i}\right\}_{i=1, \ldots, N}$ be a Langrangian basis of shape functions of $\mathbb{S}^{P}$, where $N$ is the total number of element nodes, and let $\left\{N_{i}^{f}\right\}_{i=1, \ldots, N_{f}}$ be a Lagrangian basis on the element faces, where $N_{f}$ is the total number of nodes on the element faces. Thus, $p_{w h}$, 
$S_{o_{h}}, \mathbf{q}_{p_{h}}, \mathbf{q}_{s_{h}}$, and $\hat{p}_{w_{h}}, \hat{S}_{o h}$ are defined as:

$$
\begin{aligned}
p_{w_{h}}(\mathbf{x}, t)=\sum_{i=1}^{N} p_{i}(t) N_{i}(\mathbf{x}), & S_{o_{h}}(\mathbf{x}, t)=\sum_{i=1}^{N} S_{i}(t) N_{i}(\mathbf{x}), \\
\mathbf{q}_{p_{h}}(\mathbf{x}, t)=\sum_{i=1}^{N} \sum_{j=1}^{N_{s d}} q_{p_{i, j}}(t) N_{i}(\mathbf{x}) \mathbf{e}_{j}, & \mathbf{q}_{s_{h}}(\mathbf{x}, t)=\sum_{i=1}^{N} \sum_{j=1}^{N_{s d}} q_{s i, j}(t) N_{i}(\mathbf{x}) \mathbf{e}_{j}, \\
\hat{p}_{w_{h}}(\mathbf{x}, t)=\sum_{i=1}^{N_{f}} \hat{p}_{i}(t) N_{l}^{f}(\mathbf{x}), & \hat{S}_{o_{h}}(\mathbf{x}, t)=\sum_{i=1}^{N_{f}} \hat{S}_{i}(t) N_{l}^{f}(\mathbf{x}) .
\end{aligned}
$$

Similarly, the partial derivative respect time of the oil saturation, $\dot{S}_{o_{h}}=\partial S_{o_{h}} / \partial t$, is defined as:

$$
\dot{S}_{O_{h}}(\mathbf{x}, t)=\sum_{i=1}^{N} \dot{S}_{i}(t) N_{i}(\mathbf{x})
$$

where $\dot{S}_{i}(t)=d \dot{S}_{i}(t) / d t$. By inserting Equations (16) and (17) into Equations (14) and (15), we obtain a coupled system of differential algebraic equations (DAE). In this work, we apply a high-order implicit temporal scheme to increase the accuracy of the simulation in the temporal domain.

\section{Time discretization}

We rewrite the DAE system in a compact form as:

$$
\mathbf{F}\left(t, \mathbf{S}_{o}, \dot{\mathbf{S}_{o}}, \mathbf{q}_{s}, \hat{\mathbf{S}}_{o}, \mathbf{p}_{w}, \mathbf{q}_{p}, \hat{\mathbf{p}}_{w}\right)=\mathbf{0},
$$

where $\mathbf{S}_{o}, \dot{\mathbf{S}_{o}}, \mathbf{p}_{w}, \mathbf{q}_{s}, \mathbf{q}_{p}, \hat{\mathbf{S}}_{o}$ and $\hat{\mathbf{p}}_{w}$ are the time dependent nodal values. To solve the DAE in Equation (18), we use a diagonally implicit Runge-Kutta method (DIRK). From now on, we denote by $(\cdot)^{n}$ the value of any variable at time $t^{n}$ and by $(\cdot)^{n, i}$ the value of any variable at time $t^{n, i}=t^{n}+c_{i} \Delta t$, being $n$ the time step and $i$ the DIRK stage. Thus, we compute the oil saturation at time $t^{n+1}=t^{n}+\Delta t$ as:

$$
\mathbf{S}_{o}^{n+1}=\mathbf{S}_{o}^{n}+\Delta t \sum_{i=1}^{s} b_{i} \mathbf{k}_{i},
$$

where $\mathbf{k}_{i}$ is the approximation of $\mathbf{S}_{o}$ at time $t^{n, i}$, and $s$ is the total number of stages. The oil saturation at each stage of the DIRK scheme is computed as:

$$
\mathbf{S}_{o}^{n, i}=\mathbf{S}_{o}^{n}+\Delta t \sum_{j=1}^{i} a_{i j} \mathbf{k}_{j}
$$


Table 1. Butcher's table for a diagonal implicit Runge-Kutta scheme.

$$
\begin{array}{c|cccc}
c_{1} & a_{11} & & & \\
c_{2} & a_{21} & a_{22} & & \\
\vdots & \vdots & & \ddots & \\
c_{s} & a_{s 1} & & \ldots & a_{s s} \\
\hline & b_{1} & b_{2} & \ldots & b_{s}
\end{array}
$$

and the $\mathbf{k}_{i}$ for $i=1, \ldots, s$ are computed as the solution of the non-linear algebraic equation:

$$
\mathbf{F}\left(t^{n, i}, \mathbf{S}_{o}^{n}+\Delta t \sum_{j=1}^{i} a_{i j} \mathbf{k}_{j}, \mathbf{k}_{i}, \mathbf{q}_{s}^{n, i}, \hat{\mathbf{S}}_{o}^{n, i}, \mathbf{p}_{w}^{n, i}, \mathbf{q}_{p}^{n, i}, \hat{\mathbf{p}}_{w}^{n, i}\right)=\mathbf{0}
$$

The parameters $b_{i}, c_{i}, a_{i j}$ define the DIRK method, and are given by the Butcher's tables, see Table 1, Butcher (2016); Montlaur, Fernandez-Mendez, and Huerta (2012).

\section{Non-linear solver}

To solve Equation (20), we use a fix-point iteration method. The main idea is to iteratively solve the saturation and the pressure until convergence is achieved, see Algorithm 1. To this aim, we let $l$ be the $l$-th iteration of the non-linear solver. Thus, we first solve Equation (20) for the oil saturation by imposing:

$$
\mathbf{F}(\underbrace{t^{n, i}, \mathbf{S}_{o}^{n}+\Delta t \sum_{j=1}^{i-1} a_{i j} \mathbf{k}_{j}}_{\text {Data }}+\underbrace{\Delta t a_{i i} \mathbf{k}_{i}^{l+1}, \mathbf{k}_{i}^{l+1}, \mathbf{q}_{s}^{n, i, l+1}, \hat{\mathbf{S}}_{o}^{n, i, l+1}}_{\text {Unknowns }}, \underbrace{\mathbf{p}_{w}^{n, i, l} \mathbf{q}_{p}^{n, i, l}, \hat{\mathbf{p}}_{w}^{n, i, l}}_{\text {Data }})=\mathbf{0},
$$

from which we compute $\left(\mathbf{k}_{i}^{l+1}, \mathbf{q}_{s}^{n, i, l+1}, \hat{\mathbf{S}}_{o}^{n, i, l+1}\right)$ given $\left(\mathbf{p}_{w}^{n, i, l}, \mathbf{q}_{p}^{n, i, l}, \hat{\mathbf{p}}_{w}^{n, i, l}\right)$, Line 6 of Algorithm 1. We compute $\mathbf{S}_{o}^{n, i, l+1}$ using Equation (19), Line 7 of Algorithm 1. Then, we also solve Equation (20) for the water pressure by imposing:

$$
\mathbf{F}(\underbrace{t^{n, i}, \mathbf{S}_{o}^{n}+\Delta t \sum_{j=1}^{i} a_{i j} \mathbf{k}_{j}^{l+1}, \mathbf{k}_{i}^{l+1}, \mathbf{q}_{s}^{n, i, l+1}, \hat{\mathbf{S}}_{o}^{n, i, l+1}}_{\text {Data }}, \underbrace{\mathbf{p}_{w}^{n, i, l+1}, \mathbf{q}_{p}^{n, i, l+1}, \hat{\mathbf{p}}_{w}^{n, i, l+1}}_{\text {Unknowns }})=\mathbf{0},
$$

from which we obtain $\left(\mathbf{p}_{w}^{n, i, l+1}, \mathbf{q}_{p}^{n, i, l+1}, \hat{\mathbf{p}}_{w}^{n, i, l+1}\right)$ given $\left(\mathbf{k}_{i}^{l+1}, \mathbf{q}_{s}^{n, i, l+1}, \hat{\mathbf{S}}_{o}^{n, i, l+1}\right)$, Line 8 of Algorithm 1.

This procedure will be repeated until convergence is achieved at each Runge-Kutta stage, $i=1, \ldots, s$, Line 10 of Algorithm 1. We define the stopping criteria of the 
non-linear solver as:

$$
\frac{\left\|p_{w}^{n, i, l}-p_{w}^{n, i, l+1}\right\|_{L^{2}(\Omega)}}{\left\|p_{w}^{n, i, l}\right\|_{L^{2}(\Omega)}}<\varepsilon_{p_{w}}, \quad \frac{\left\|S_{o}^{n, i, l}-S_{o}^{n, i, l+1}\right\|_{L^{2}(\Omega)}}{\left\|S_{o}^{n, i, l}\right\|_{L^{2}(\Omega)}}<\varepsilon_{S_{o}},
$$

where $\varepsilon_{p_{w}}$ and $\varepsilon_{S_{o}}$ are two prescribed tolerances.

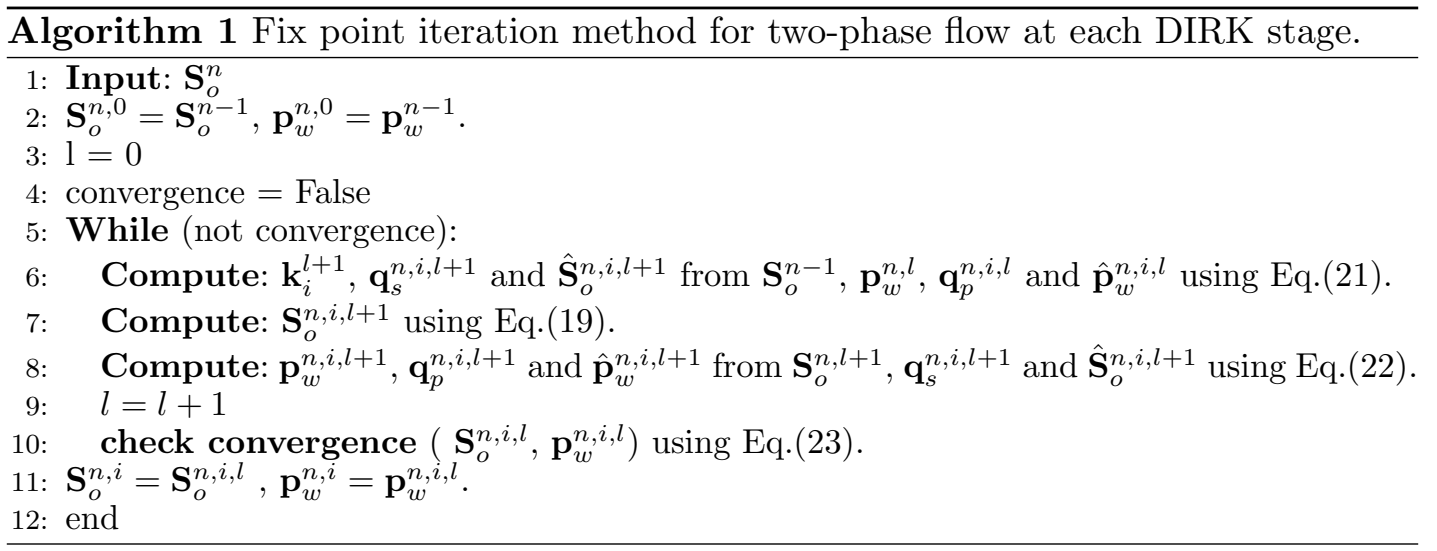

We highlight that for each iteration of the fix-point method we need to solve two linear systems, one for the saturation, Equation (21) and another for the pressure, Equation (22). Each system to be solved is hybridized in order to solve a linear system for $\hat{\mathbf{S}}_{o}^{n, i, l+1}$ and another for $\hat{\mathbf{p}}_{w}^{n, i, l+1}$. The other unknowns are recovered using an element-wise process.

The proposed method differs from the classical and widely used IMPES method to solve two-phase flow problem, see Chen, Huan, and Ma (2006). IMPES method solves the pressure implicitly and the saturation explicitly, whereas our formulation solves both variables implicitly. The implicit scheme allows using larger time steps. Moreover, the iterative method detailed in Algorithm 1 allows obtaining high-order accurate approximations.

\section{Local post-processing}

One of the main advantages of using the HDG formulation is that the scalar variables (pressure and saturation), and their fluxes, in $\mathbb{V}_{h}^{P}$ and $\mathbf{W}_{h}^{P}$ spaces, respectively, have a rate of convergence of $P+1$ in the $L^{2}$-norm, when the temporal error is low enough. Moreover, a local post-processing can be applied to obtain a new approximation for the pressure, $p_{w_{h}}^{*}$, and for the saturation, $S_{o_{h}}^{*}$, both in $\mathbb{V}_{h}^{P+1}$ with convergence rate of $P+2$ in the $L^{2}$-norm for diffusion dominated problems, see Kirby, Sherwin, and Cockburn (2012).

In our formulation, we have two local problems. The first one consists on finding the post-processed pressure, $p_{w_{h}}^{*} \in \mathbb{V}_{h}^{P+1}$ on each element, $e$, such that:

$$
\begin{aligned}
\left(\mathbf{K} \lambda_{t} \boldsymbol{\nabla} p_{w_{h}}^{*}, \boldsymbol{\nabla} v\right)_{e} & =-\left(\mathbf{q}_{p_{h}}, \boldsymbol{\nabla} v\right)_{e} \\
\left(p_{w_{h}}^{*}, 1\right)_{e} & =\left(p_{w_{h}}, 1\right)_{e},
\end{aligned}
$$

for all $v \in \mathbb{V}_{h}^{P+1}$. The second local problem consist on finding the post-processed 
Table 2. Butcher's table for a DIRK3-s3 scheme.

$$
\begin{array}{c|ccc}
\begin{array}{c}
\gamma \\
\frac{1+\gamma}{2}
\end{array} & \frac{1-\gamma}{2} & \gamma \\
1 & \frac{-6 \gamma^{2}+16 \gamma-1}{4} & \frac{6 \gamma^{2}-20 \gamma-1}{4} & \gamma \\
\hline & \frac{-6 \gamma^{2}+16 \gamma-1}{4} & \frac{6 \gamma^{2}-20 \gamma-1}{4} & \gamma
\end{array}
$$

saturation, $S_{o_{h}}^{*} \in \mathbb{V}_{h}^{P+1}$ on each element, e, such that:

$$
\begin{aligned}
\left(\mathbf{K} \lambda_{o} p_{c}{ }^{\prime} \nabla S_{o_{h}}^{*}, \nabla v\right)_{e} & =-\left(\mathbf{q}_{s_{h}}, \nabla v\right)_{e} \\
\left(S_{o_{h}}^{*}, 1\right)_{e} & =\left(S_{o_{h}}, 1\right)_{e} .
\end{aligned}
$$

According to Kirby, Sherwin, and Cockburn (2012) it is important to highlight that this procedure can be applied at selected time steps, and it is not necessary to apply it at all the time steps.

\section{Results}

In this section, we first validate the proposed formulation and the applied methodology by showing numerical evidence of the convergence rates. Second, we apply our method to simulate two applications of water-flooding technique for oil recovery. Specifically, in the second example, we solve the classical five spot pattern problem, and in the third one, we consider an heterogeneous domain.

In all the examples, we use the diagonally implicit Runge-Kutta method of order three with three stages, DIRK3-s3, for the time discretization. The Runge-Kutta parameters are specified in Table 2, setting $\gamma=0.4358665215$, Butcher (2016).

In the last two examples, we use the Brooks-Corey model, Equation (4), with $p_{e}=$ $10^{3} \mathrm{~Pa}, \theta=2, S_{r w}=0.2$ and $S_{r o}=0.2$. Similarly, in those examples we apply the following boundary conditions:

$$
\begin{array}{lll}
p_{w}=3 \cdot 10^{6} \mathrm{~Pa}, & S_{o}=g_{D s}^{i n}, & \text { on } \Gamma_{\text {in }} \\
p_{w}=10^{6} \mathrm{~Pa}, & \left(\frac{\lambda_{o} \lambda_{w}}{\lambda_{t}} \mathbf{K} \boldsymbol{\nabla} p_{c}\right) \cdot \mathbf{n}=0, & \text { on } \Gamma_{\text {out }} \\
\mathbf{u}_{t} \cdot \mathbf{n}=0, & \mathbf{u}_{o} \cdot \mathbf{n}=0, & \text { on } \Gamma_{n f},
\end{array}
$$

where $g_{D s}^{i n}$ will be different in each example. The initial condition for the saturation is $S_{o}(\cdot, 0)=1.1 S_{r o}$. We set the characteristic lengths in Equations (12) and (13) as $l_{p}=10^{-6}$ and $l_{s}=10^{-10}$, and the tolerances of the non-linear solver, Equation (23), as $\varepsilon_{p_{w}}=10^{-4}$ and $\varepsilon_{S_{o}}=10^{-5}$.

\subsection{Convergence rate analysis}

In this example, we show numerical evidence of the convergence rates for the $p_{w_{h}}, S_{o_{h}}$, $\mathbf{q}_{p_{h}}, \mathbf{q}_{s_{h}}$ and the post-processed pressure and saturation, $p_{w_{h}}^{*}$ and $S_{o_{h}}^{*}$, respectively, of our formulation. This example serves as a validation of our formulation and the proposed methodology. 
We define an analytical solution for the water pressure, $p_{w}$, and the oil saturation, $S_{o}$, as:

$$
\begin{aligned}
& p_{w}=0.5+t\left(\frac{\cos (\pi x) \cos (\pi y)}{4}\right), \\
& S_{o}=0.5+t\left(\frac{\sin (\pi x) \sin (\pi y)}{4}\right),
\end{aligned}
$$

where $(x, y) \in \Omega=[0,1] \mathrm{m} \times[0,1] \mathrm{m}$ and $t \in(0,1) \mathrm{s}$. We prescribe Dirichlet boundary condition for the water pressure and oil saturation, and the source terms are taken to satisfy the PDEs with the proposed analytical solutions. We set the soil permeability as $\mathbf{K}=\mathbf{I} \mathrm{m}^{2}$, the porosity as $\phi=0.1$, the oil viscosity as $\mu_{o}=1 \mathrm{~Pa} \cdot \mathrm{s}$ and the water viscosity as $\mu_{w}=0.1 \mathrm{~Pa} \cdot \mathrm{s}$. Specifically for this example, we use the Brooks-Corey model, Equation (4), with $p_{e}=0.5 \mathrm{~Pa}, \theta=1$. We select $\tau_{p}=1$ and $\tau_{s}=10$, see Equations (12) and (13).

To study the convergence rates, we discretize $\Omega$ using quadrilateral meshes composed between 9 to 6400 elements of polynomial degree from 1 to 3 . Moreover, we use a DIRK3-s3 scheme with $\Delta t=0.25 \mathrm{~s}$ for the time discretization. At $t=1 \mathrm{~s}$, we measure the error in the $L^{2}$-norm of the numerical solutions against the analytical ones.

Figure 1 shows the convergence rates of the pressure and saturation, their fluxes, and the post-processed solutions. We obtain the expected convergence rate of $P+1$ in $L^{2}$-norm for the water pressure, the oil saturation and for the fluxes $\mathbf{q}_{p}$ and $\mathbf{q}_{s}$. The local post-process, detailed in Equations (24) and (25), is applied to obtain a super convergence rate of $P+2$ in $L^{2}$-norm of the post-processed pressure, $p_{w_{h}}^{*}$, and the post-processed saturation, $S_{o_{h}}^{*}$.

This example shows two main advantages of using HDG formulation, which are the optimal convergence rate of $P+1$ in $L^{2}$-norm for the solutions and their fluxes, and the convergence rate of $P+2$ in $L^{2}$-norm for the post-processed solutions.

\subsection{Water-flooding in five spot pattern}

We consider a square domain, $\Omega$, of $140 \mathrm{~m} \times 140 \mathrm{~m}$. The selected pattern has four injection wells located at the vertices of square, and one producer well at its center, see Figure 2. The radius of the wells is $r_{w}=5 \mathrm{~m}$. On the boundary $\partial \Omega=\Gamma_{\text {in }} \bigcup \Gamma_{\text {out }} \bigcup \Gamma_{n f}$, we apply the boundary conditions detailed in Equation (26), with $g_{D s}^{i n}=0.3$. The soil permeability is $\mathbf{K}=10^{-11} \mathbf{I} \mathrm{m}^{2}$, the porosity is $\phi=0.2$, and the viscosity for the water and oil phases are $\mu_{w}=0.001 \mathrm{~Pa} \cdot \mathrm{s}$ and $\mu_{o}=0.01 \mathrm{~Pa} \cdot \mathrm{s}$, respectively.

We discretize $\Omega$ with 930 triangular elements of polynomial degree three (4314 nodes), see Figure 2. The number of unknowns involved in the linear systems that have to be solved in each iteration of Equations (21) and (22) is 33652. Nevertheless, applying a static condensation procedure in the HDG formulation it is reduced to 5752 unknowns for each one. The time step for the DIRK3-s3 scheme is $\Delta t=900 \mathrm{~s}$.

Figure 3 shows the water pressure and saturation approximations at time $t=51 \mathrm{~h}$ and $t=65 \mathrm{~h}$. At the initial stage the water saturation, $S_{w}$, is equal to the residual water saturation, $S_{w}(\cdot, 0)=1-S_{o}(\cdot, 0)$, and oil occupies the rest of the voids. Afterwards, water is injected from the corner wells (injectors), moving the oil to the pumping well at the center, and occupies the space left by the oil. This is observed in Figures 3(a) and $3(\mathrm{~b})$, in which we show the water saturation and the water velocity vectors at time $t=51 \mathrm{~h}$ and $t=65 \mathrm{~h}$, respectively. The water saturation increases from the 


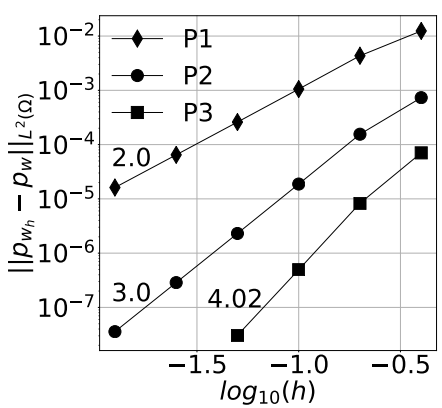

(a)

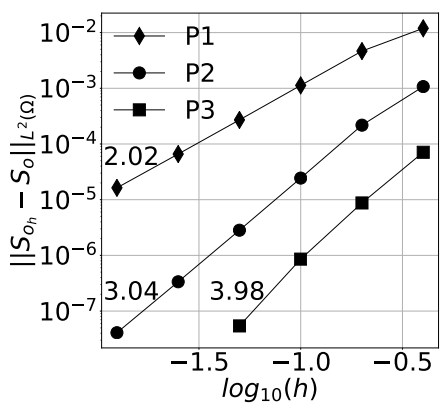

(d)

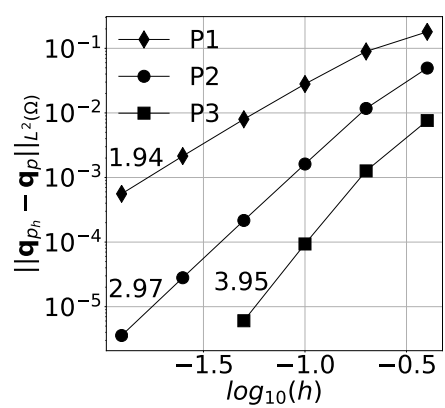

(b)

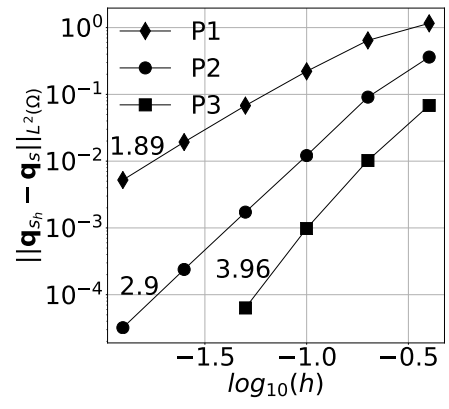

(e)

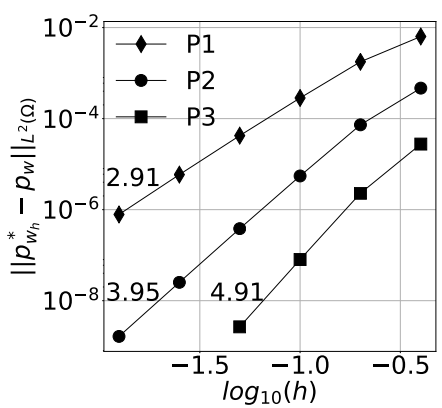

(c)

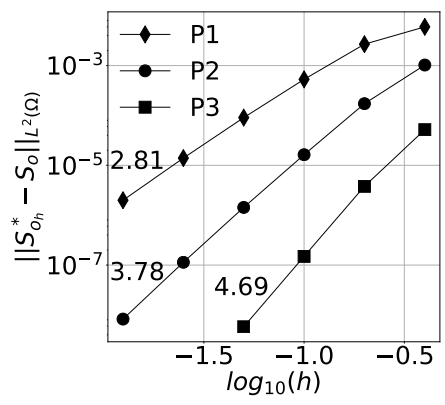

(f)

Figure 1. Convergence rates for: a) water pressure; b) water pressure flux, c) post-processed water pressure, d) oil saturation, e) oil saturation flux, and f) post-processed oil saturation.

injectors wells to the producer well, as the result of mobilising the oil to the producer well. Moreover, the water moves away from injector wells, while it moves towards the producer well. Figures 3(c) and 3(d), show the water pressure field. As expected, the water pressure has higher values at the injector wells and lower values at the extractor well.

\subsection{Water-flooding in heterogeneous material}

In this example, we consider a domain composed of two different permeability regions, Figure 4. The simulation domain, $\Omega$, is a square of $100 \mathrm{~m} \times 100 \mathrm{~m}$, and its boundary is $\partial \Omega=\Gamma_{\text {in }} \bigcup \Gamma_{\text {out }} \bigcup \Gamma_{n f}$, see Figure 4 . We prescribe the boundary conditions detailed in Equation (26) with $g_{D s}^{i n}=0.22$. The soil permeability is $\mathbf{K}_{A}=10^{-13} \mathbf{I} \mathrm{m}^{2}$ and $\mathbf{K}_{B}=10^{-12} \mathbf{I} \mathrm{m}^{2}$, the porosity is $\phi=0.2$ and the viscosity for the water and oil phases are $\mu_{w}=0.001 \mathrm{~Pa} \cdot \mathrm{s}$ and $\mu_{o}=0.012 \mathrm{~Pa} \cdot \mathrm{s}$, respectively.

We discretize the domain using 256 quadrilateral elements of polynomial degree four (4225 nodes), see Figure 4. The number of unknowns involved in the linear systems that have to be solved in each iteration of Equations (21) and (22) is 21920. Nevertheless, applying a static condensation procedure in the HDG formulation it is reduced to 2720 unknowns for each one. The time step for the DIRK3-s3 scheme is $\Delta t=6 \mathrm{~h}$.

Figure 5 shows the computed water saturation and water pressure at time $t=30$ days and $t=100$ days. In Figures $5(\mathrm{a})$ and $5(\mathrm{~b})$, we show the water saturation and the water velocity vectors. At the beginning, the oil saturates homogeneously the porous media. Then, water is injected along the inflow boundary and moves the oil towards the outflow boundary. At both time steps the water is moving faster within the bottom 


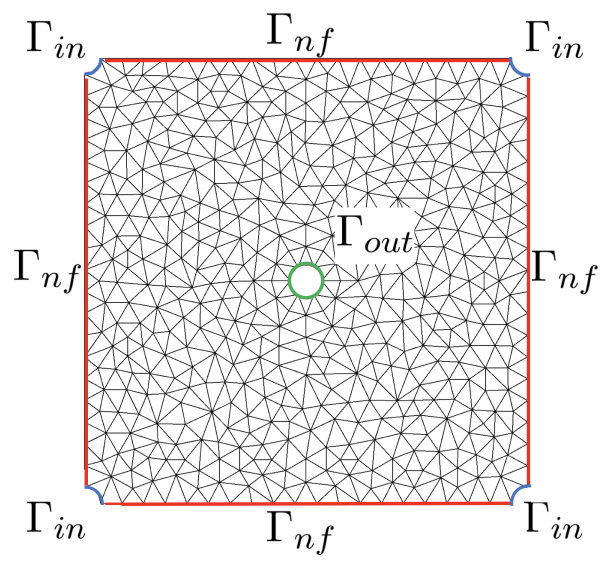

Figure 2. Mesh and boundary conditions distributions for the five spot example

region, where the permeability is higher, than in the upper region. Figures 5(c) and $5(\mathrm{~d})$, show the water pressure field at the same time steps. Highest water pressure values are on the inflow boundary and the lowest on the outflow boundary. Moreover, when the injected water reaches to the outflow boundary, the water pressure in the entrance is equalized in both regions, and the water starts to saturate the upper region, as can be observed in Figures 5(b) and 5(d).

\section{Conclusions and future work}

In this work, a hybridizable discontinuous Galerkin method has been developed to solve the two-phase flow in porous media problem. To this end, we have rewritten the initial second order PDEs as a set of first-order PDEs, and the weak form of the problem has been deduced in terms of the traces of the water pressure and oil saturation. The stabilization parameters are computed in terms of the oil and total phase mobilities and in terms of the maximum eigenvalue of the permeability matrix. We provide a framework to use high-order time discretization with a fix-point iteration method to compute the saturation and the pressure implicitly. This differs from the classical IMPES method, in which the saturation is computed explicitly.

In the proposed method, we use a Lagrangian basis of shape functions to define the elemental polynomial spaces and therefore, the unknowns of the problem are the nodal values. Other bases of the polynomial space could be used, such as orthonormal polynomials. Thus, the unknowns of the problem would be the coefficients of the polynomial expansion of the solution in the used basis. Nevertheless, in our work, we selected the Lagrangian basis, since we use a non-uniform nodal distribution that approximatively minimizes the Lebesgue constant, see Warburton (2006). The used basis is well-suited for high-order Lagrange interpolation. Moreover, the solution of the problem are the nodal values, which is convenient for practical purposes.

The proposed formulation has been applied to solve several problems. First, a synthetic example with analytical solution is considered, and expected convergence rates for the solutions, their fluxes and the post-processed approximations are validated. Second, our formulation has been applied to solve the water-flooding technique for oil recovery simulation. Specifically, to illustrate the capabilities of the proposed method, we present two examples considering several wells and heterogeneous reservoirs. 


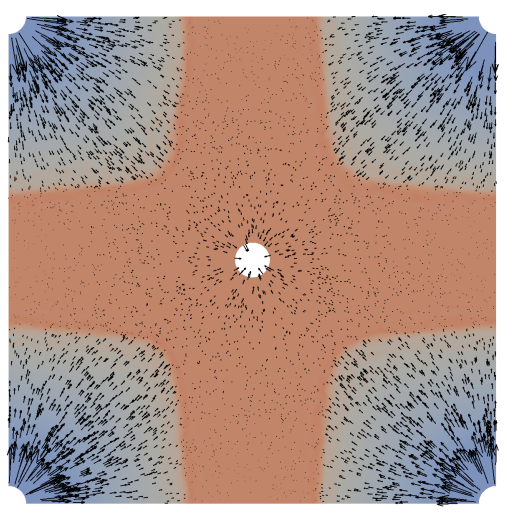

(a)

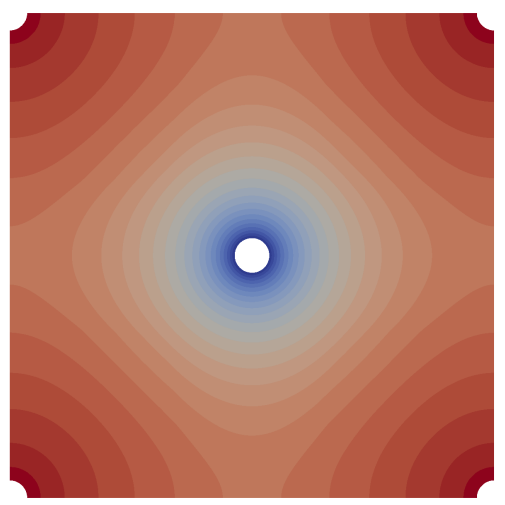

(c)

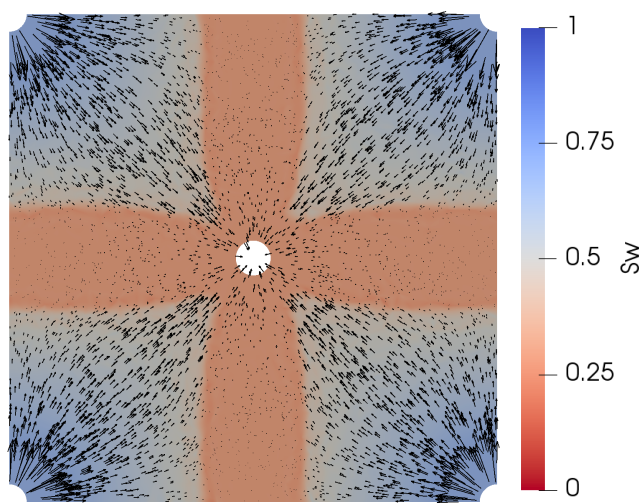

(b)

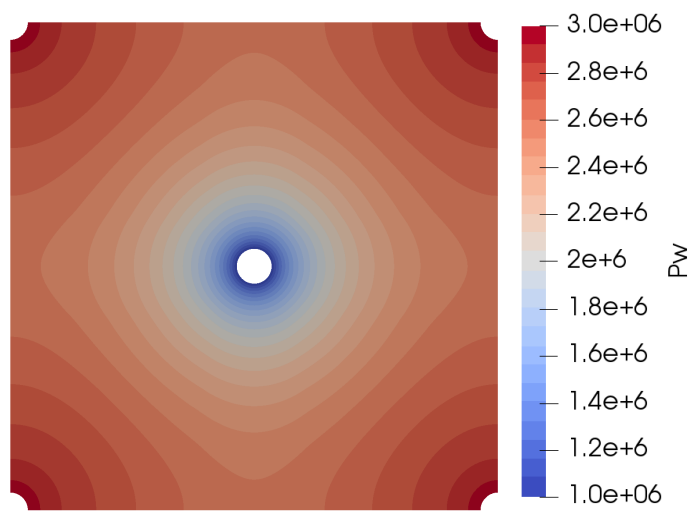

(d)

Figure 3. Water saturation approximation at time: a) 51 hours, and b) 65 hours. Water pressure approximation at time: c) 51 hours, and d) 65 hours.

Several aspects of this work will be analyzed and improved in the near future. First, an alternative non-linear solver will be developed to improve the computational efficiency. Second, we will extend our HDG formulation to 3D problems considering slightly compressible fluids. Third, we will compare the influence of the capillary pressure models by comparing the solutions with other models, such as the Van Genuchten model. Fourth, we will analyse a dimensionless formulation of our method in order to reduce the computational cost and increase the accuracy of the approximations. Finally, parallel implementation of the proposed method will be developed in order to apply it to large oil reservoirs.

\section{Acknowledgments}

This work has been supported by FEDER and the Spanish Government, "Ministerio de Economía y Competitividad" grant project contract CTM2014-55014-C3-3-R, "Ministerio de Ciencia Innovación y Universidades" grant project contract PGC2018097257-B-C33, and the grant BES-2015-072833. 


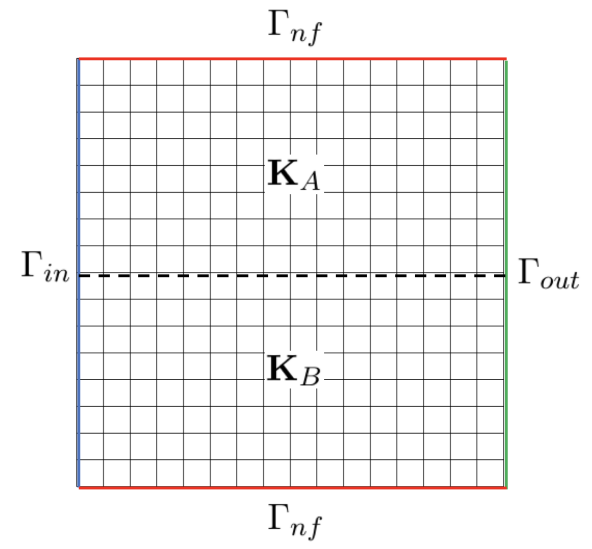

Figure 4. Mesh and boundary conditions distributions for the heterogeneous material example

\section{References}

Bear, J., and A. Verruijt. 2012. Modeling groundwater flow and pollution. Vol. 2. Springer Science \& Business Media.

Butcher, J.C. 2016. Numerical methods for ordinary differential equations. John Wiley \& Sons.

Chen, Z., G. Huan, and Y. Ma. 2006. Computational methods for multiphase flows in porous media. Vol. 2. Siam.

Corey, A.T. 1964. "Hydraulic properties of porous media." Colorade State University, Hydraulic Papers (3).

Donaldson, E.C., G.V. Chilingarian, and T.F. Yen. 1985. Enhanced oil recovery, I: fundamentals and analyses. Elsevier.

Epshteyn, Y., and B. Rivière. 2007. "Fully implicit discontinuous finite element methods for two-phase flow." Applied Numerical Mathematics 57 (4): 383-401.

Kirby, R.M., S.J. Sherwin, and B. Cockburn. 2012. "To CG or to HDG: a comparative study." Journal of Scientific Computing 51 (1): 183-212.

Klieber, W., and B. Rivière. 2006. "Adaptive simulations of two-phase flow by discontinuous Galerkin methods." Computer Methods in Applied Mechanics and Engineering 196 (1-3): 404-419.

Montlaur, A, S. Fernandez-Mendez, and A. Huerta. 2012. "High-order implicit time integration for unsteady incompressible flows." International journal for numerical methods in fluids 70 (5): 603-626.

Nguyen, N.C., J. Peraire, and B. Cockburn. 2009a. "An implicit high-order hybridizable discontinuous Galerkin method for linear convection-diffusion equations." Journal of Computational Physics 228 (9): 3232-3254.

Nguyen, N.C., J. Peraire, and B. Cockburn. 2009b. "An implicit high-order hybridizable discontinuous Galerkin method for nonlinear convection-diffusion equations." Journal of Computational Physics 228 (23): 8841-8855.

Nguyen, N.C., J. Peraire, and B. Cockburn. 2011. "High-order implicit hybridizable discontinuous Galerkin methods for acoustics and elastodynamics." Journal of Computational Physics 230 (10): $3695-3718$.

Sevilla, R., and A. Huerta. 2016. "Tutorial on Hybridizable Discontinuous Galerkin (HDG) for second-order elliptic problems." In Advanced Finite Element Technologies, 105-129. Springer.

Warburton, T. 2006. "An explicit construction of interpolation nodes on the simplex." Journal of engineering mathematics 56 (3): 247-262. 


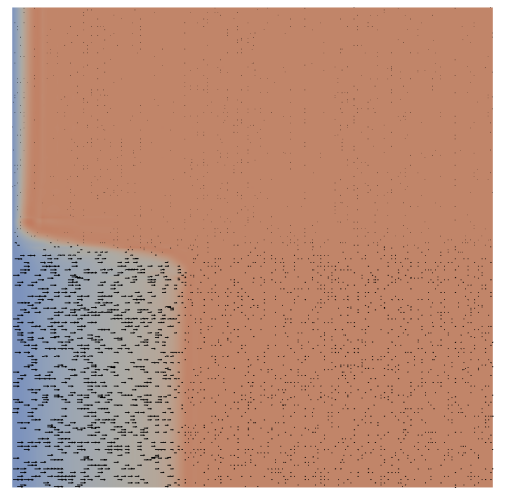

(a)

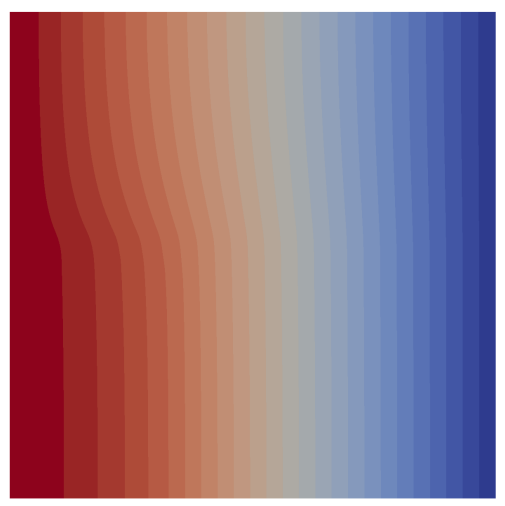

(c)

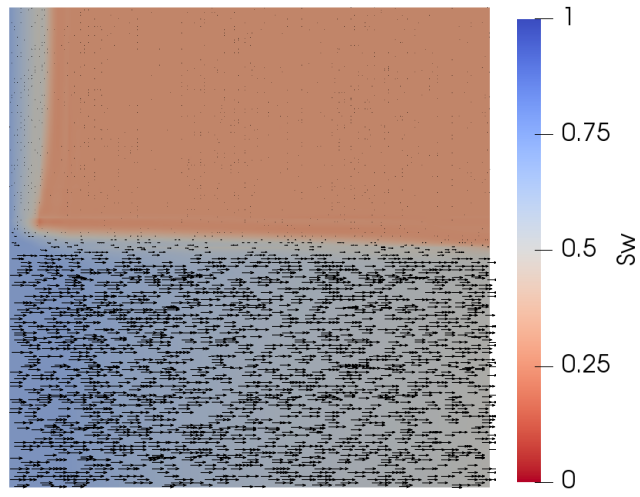

(b)

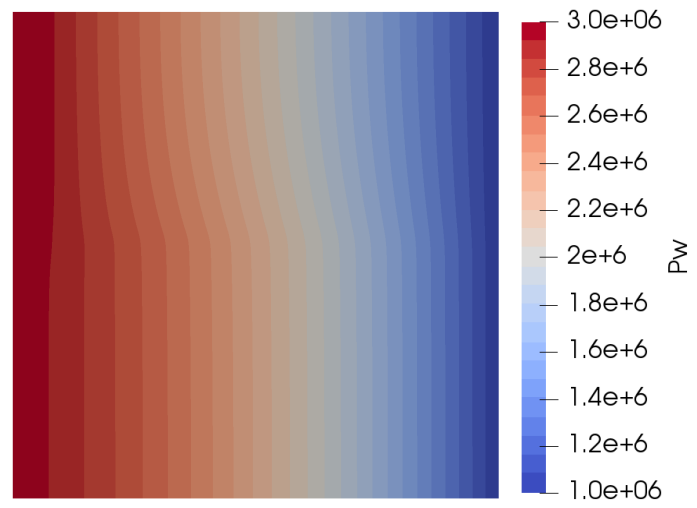

(d)

Figure 5. Water saturation approximation at time: a) 30 days, and b) 100 days. Water pressure approximation at time: c) 30 days, and d) 100 days. 